\title{
Data scrutiny in IUCr journals
}

\author{
Brian McMahon \\ International Union of Crystallography, Chester, United Kingdom; \\ bm@iucr.org
}

Peer review of supporting data for submitted research articles is currently assuming great significance in scientific publishing, but is not new for the journals of the IUCr. Co-editors of Acta Crystallographica C under the editorship of Sidney Abrahams (1924-2021) were expected to validate the consistency of crystal structure data for reported structures, for which cell parameters and symmetry, coordinates, geometry and anisotropic displacement parameters were mandatory. The journal developed software to reduce the calculational burden, and this evolved into the checkCIF service that allowed authors to participate in the validation effort, and to account for apparent anomalies or outliers in their derived structures. An early consequence was the almost complete elimination of corrigenda that resulted from post-publication surveys by individual scientists or by database aggregators. Over the years checkCIF increased in sophistication and power (authors were required to supply structure factors in machine-readable form), and has been adopted by other journal publishers and by structural databases. The IUCr Diffraction Data Deposition Working Group (2011-2017) emphasised the value of access to raw experimental data in evaluating structure interpretation, and IUCr Journals have responded by encouraging authors to make available their diffraction data sets. The journals continue to explore ways to improve the refereeing process with regard to data, in their effort to make the initial publication of the version of record of an article as error-free as possible.

Keywords: data validation, peer review, publishing, checkCIF, CIF 